\title{
Standards for Neurologic Critical Care Units: A Statement for Healthcare Professionals from The Neurocritical Care Society
}

\author{
Asma M. Moheet ${ }^{*}$, Sarah L. Livesay ${ }^{2}$, Tamer Abdelhak ${ }^{3}$,Thomas P. Bleck² ${ }^{2}$ Theresa Human ${ }^{4}$, Navaz Karanjia ${ }^{5}$, \\ Amanda Lamer-Rosen ${ }^{1}$, Joshua Medow ${ }^{6}$, Paul A. Nyquist ${ }^{7}$, Axel Rosengart ${ }^{1}$, Wade Smith ${ }^{8}$, Michel T. Torbey ${ }^{9}$ \\ and Cherylee W. J. Chang ${ }^{10}$
}

(c) 2018 Springer Science+Business Media, LLC, part of Springer Nature and Neurocritical Care Society

\begin{abstract}
Neurocritical care is a distinct subspecialty focusing on the optimal management of acutely ill patients with lifethreatening neurologic and neurosurgical disease or with life-threatening neurologic manifestations of systemic disease. Care by expert healthcare providers to optimize neurologic recovery is necessary. Given the lack of an organizational framework and criteria for the development and maintenance of neurological critical care units (NCCUs), this document is put forth by the Neurocritical Care Society (NCS). Recommended organizational structure, personnel and processes necessary to develop a successful neurocritical care program are outlined. Methods: Under the direction of NCS Executive Leadership, a multidisciplinary writing group of NCS members was formed. After an iterative process, a framework was proposed and approved by members of the writing group. A draft was then written, which was reviewed by the NCS Quality Committee and NCS Guidelines Committee, members at large, and posted for public comment. Feedback was formally collated, reviewed and incorporated into the final document which was subsequently approved by the NCS Board of Directors.
\end{abstract}

Keywords: Quality, Quality indicator, Quality improvement, Performance improvement, Standards, Consensus, Neurocritical care, Neurocritical care unit, Intensive care, Intensive care unit, Critical care, Critical care unit

\section{Introduction and Background}

Neurocritical care is a distinct subspecialty focusing on the optimal management of acutely ill patients with lifethreatening neurologic and neurosurgical disease or with life-threatening neurologic manifestations of systemic disease. Significant advances in the acute treatment of neurologic disease in the past three decades necessitate care by expert healthcare providers to optimize neurologic recovery. The formation of the Neurocritical Care Society (NCS) in 2002 and the establishment of formal training in neurocritical care with physician subspecialty

\footnotetext{
*Correspondence: asma.moheet@csmc.edu

${ }^{1}$ Cedars-Sinai Medical Center, Los Angeles, CA, USA

Full list of author information is available at the end of the article

Asma M. Moheet and Sarah L. Livesay share first authorship.
}

certification and the continued development of the specialty for nurses, pharmacists and other healthcare providers, have produced a critical mass of highly-trained professionals with expertise in the management of critically ill patients with neurologic disease $[1,2]$. A growing body of evidence demonstrates that care provided by this subspecialty team improves outcomes [3-5]. However, the field lacks an organizational framework and criteria for the development and maintenance of neurological critical care units (NCCUs). As neurocritical care is an essential component of a comprehensive neurosciences program and plays a key role in the delivery of care within certified stroke and trauma programs, the NCS will outline in this paper standards to guide the development and operationalization of a NCCU by delineating the recommended organizational structure, personnel 
Table 1 Common neurocritical care diagnoses

\begin{tabular}{l}
\hline Ischemic stroke \\
Intracerebral hemorrhage \\
Subarachnoid hemorrhage \\
Acute nontraumatic weakness \\
Traumatic brain and spine injury including epidural and subdural hematoma, diffuse axonal injury \\
Anoxic brain injury \\
Coma \\
Intracranial hypertension \\
Meningitis and encephalitis \\
Status epilepticus
\end{tabular}

and processes necessary to develop a successful neurocritical care program.

\section{Methods}

Under the direction of NCS Executive Leadership, a multidisciplinary national writing group of NCS members was formed in June of 2015 to identify resources and standards by which to designate adult NCCUs in the United States. Over the next 2 years, through a series of conference calls and e-mail communications, a framework for this statement was proposed. The framework was approved by members of the writing group and a draft was then written. This draft was reviewed by the NCS Quality Committee and then submitted to the NCS Guidelines Committee and 12 members at large for formal review. After these comments were reviewed, appropriate clarifications and requisite changes were made. The document was posted on the NCS website for public commentary. Comments were formally collated, reviewed and incorporated into the final document which was subsequently approved by the NCS Board of Directors.

\section{Levels of Neurocritical Care Units}

Neurocritical care is provided to patients with a variety of diagnoses (see Table 1, Common Neurocritical Care Diagnoses) with different levels of service in a range of settings. However, a minimum level of expertise and resource investment is essential for the development of a NCCU. Factors such as geography, resource availability, and access to subspecialty personnel may influence an institution's ability to provide care for the entire epoch of the patient's illness or necessitate the transfer of a patient to a higher level of care. Prior documents in the general critical care literature have proposed level-based classification of intensive care units (ICU) [6,7]. For the purposes of this paper, we recognize three levels of NCCUs. Level I units are receiving centers for patients with complex neurological emergencies who require advanced interventions and provide the most comprehensive neurocritical care. These units should be equipped to provide definitive and expert care to a wide variety of neurocritical care disorders using an interdisciplinary approach. Level I units offer a full complement of advanced monitoring, surgical and medical treatments and have the capability to provide physician fellowship and advanced practice professional training and as such, are often associated with an academic program. Level II units can stabilize acutely ill patients and safely manage stable neurocritical disease processes, while having established relationships with Level I neurocritical care units. Level III units can provide emergent evaluation and stabilization of patients presenting with neurological emergencies and facilitate transfer of these patients to Level I and Level II units when appropriate. Clearly delineated protocols and processes are necessary to identify patients for rapid referral and safe transfer once stable for advanced subspecialty care. While a variety of systems to stratify stroke and trauma centers exist and geographical and economic factors may shape services offered, the system of stratification proposed here recognizes that high-quality neurocritical care services may be delivered in units without advanced research, academic or surgical programs and allows for flexibility in developing a program to meet the needs of the patients of any region. See Table 2 for an overview of recommendations by program level.

\section{General Organization and Infrastructure}

NCCUs, as with any ICU, should be in geographically distinct areas of a medical center with beds cohorted by specialty nursing training and allocated specifically to patients with neurocritical care diagnoses as those listed in Table 1. Proximity of the NCCU to the Emergency Department, Operating Rooms, and Imaging Departments is preferred to facilitate safe and rapid transfer of patients between these areas when required. Level I and Level II units should possess the ability to transfer and accept patients via a designated ambulance bay and/ 
Table 2 Neurocritical care unit recommendations

\begin{tabular}{|c|c|c|c|}
\hline Standards & Level I & Level II & Level III \\
\hline \multicolumn{4}{|l|}{ Organization } \\
\hline Neuroscience critical care (NCC) Service oversight by physician, nursing and hospital executive leadership & $\mathrm{R}$ & $\mathrm{R}$ & $\mathrm{R}$ \\
\hline Delineation of physician and non-physician privileges & $\mathrm{R}$ & $\mathrm{R}$ & $\mathrm{R}$ \\
\hline Distinct administrative unit & $\mathrm{R}$ & R & O \\
\hline Leadership meet regularly to evaluate service needs & $\mathrm{R}$ & $\mathrm{R}$ & $\mathrm{R}$ \\
\hline Leadership meet regularly to evaluate service needs & $\mathrm{R}$ & R & $\mathrm{R}$ \\
\hline NCC Committee & $\mathrm{R}$ & R & $\mathrm{O}$ \\
\hline Standing committee, interdisciplinary representation & $\mathrm{R}$ & $\mathrm{R}$ & O \\
\hline Unit oversight by physician, nursing and hospital executive leadership & $\mathrm{R}$ & R & $\mathrm{R}$ \\
\hline \multicolumn{4}{|l|}{ Policies/Guidelines: } \\
\hline Admit/discharge & $\mathrm{R}$ & $\mathrm{R}$ & $\mathrm{R}$ \\
\hline Determination of death by neurologic criteria & R & R & $\mathrm{R}$ \\
\hline Disease specific protocols & $\mathrm{R}$ & $\mathrm{R}$ & O \\
\hline Equipment and procedures related to NCC & $\mathrm{R}$ & R & $\mathrm{R}$ \\
\hline Equipment maintenance & $\mathrm{R}$ & $\mathrm{R}$ & $\mathrm{R}$ \\
\hline Essential equipment list & $\mathrm{R}$ & R & R \\
\hline Nosocomial infection & $\mathrm{R}$ & $\mathrm{R}$ & $\mathrm{R}$ \\
\hline Patient isolation & R & R & $\mathrm{R}$ \\
\hline Patient monitoring & $\mathrm{R}$ & $\mathrm{R}$ & $\mathrm{R}$ \\
\hline Periodic review of morbidity/mortality & $\mathrm{R}$ & $\mathrm{R}$ & $\mathrm{R}$ \\
\hline Quality & $\mathrm{R}$ & $\mathrm{R}$ & $\mathrm{R}$ \\
\hline Safety & $\mathrm{R}$ & R & $\mathrm{R}$ \\
\hline System of record-keeping & $\mathrm{R}$ & R & R \\
\hline Traffic control & $\mathrm{R}$ & R & $\mathrm{R}$ \\
\hline Transfer & $\mathrm{R}$ & $\mathrm{R}$ & R \\
\hline Visitation and family/surrogate-integrated care & $\mathrm{R}$ & $\mathrm{R}$ & $\mathrm{R}$ \\
\hline
\end{tabular}

\section{Medical director}

Appointed by appropriate hospital authority

Acknowledged in writing

Name qualified physician to fulfill duties when unavailable, communicated to interdisciplinary team

Written documentation of responsibilities

\section{Medical director qualifications:}

Neurocritical Care subspecialty (board) certified or eligible $\quad R$

Assure policy implementation

Coordinate research

Ensure staff education

Maintain database or vital statistics

Participate in budget preparation

Participates in development, review and implementation of policies

Quality oversight of any NCC patient

Supervise quality improvement (QI) and quality assurance (QA) activities

Supervise resuscitation techniques

$\begin{array}{lll}R & R & R \\ R & R & R \\ R & R & R \\ R & R & R\end{array}$

Physician staff or Licensed Independent Practitioner (LIP)

Certified Neurointensivist or LIP or post graduate year 2 or above with NCC expertise and neurointensivist support:

Available in-house $24 \mathrm{~h}$ a day

Available at bedside* within 5 min $24 \mathrm{~h}$ day

Available by telemedicine or telephone

All physicians should have subspecialty certification in NCC

Physicians should have subspecialty training in NCC

All medical staff undergo OPPE case review in NCC (or equivalent)

$\begin{array}{lll}R & R & O \\ R & R & R \\ R & O & O \\ R & R & R \\ R & R & R \\ R & R & R \\ R & R & R \\ R & R & R \\ R & R & R \\ R & R & R\end{array}$

\begin{tabular}{lll}
$R$ & $O$ & $O$ \\
$R$ & $R$ & $O$ \\
$R$ & $R$ & $R$ \\
$R$ & $O$ & $O$ \\
$R$ & $R$ & $R$ \\
$R$ & $R$ & $O$ \\
\hline
\end{tabular}


Table 2 continued

\begin{tabular}{|c|c|c|c|}
\hline Standards & Level I & Level II & Level III \\
\hline Additional privileges in NCC determined by organization & $\mathrm{R}$ & $\mathrm{R}$ & $\mathrm{R}$ \\
\hline Neurointensivist on-site 24/7. May be delegated to another in-house provider (trainee, advanced practice provider) & $\mathrm{R}$ & O & $\mathrm{O}$ \\
\hline When off-site, neurointensivist must return calls within 5 min, at bedside within 5 min* & $\mathrm{R}$ & $\mathrm{R}$ & $\mathrm{O}$ \\
\hline \multicolumn{4}{|l|}{ Available in less than $30 \mathrm{~min}$ : } \\
\hline Neurointerventionalist & $\mathrm{R}$ & $\mathrm{O}$ & $\mathrm{O}$ \\
\hline Neurosurgeon & $\mathrm{R}$ & $\mathrm{O}$ & $\mathrm{O}$ \\
\hline Radiologist & $\mathrm{R}^{*}$ & $\mathrm{R}^{*}$ & $\mathrm{R}^{*}$ \\
\hline \multicolumn{4}{|l|}{ Available in less than $1 \mathrm{~h}$ : } \\
\hline Neurointerventionalist & $\mathrm{R}$ & $\mathrm{R}^{* *}$ & $\mathrm{O}$ \\
\hline Neurosurgeon & R & $R^{* *}$ & 0 \\
\hline
\end{tabular}

Available for consultation:

\begin{tabular}{|c|c|c|c|}
\hline Anesthesiologist & $\mathrm{R}$ & $\mathrm{R}$ & $\mathrm{O}$ \\
\hline Cardiologist & $\mathrm{R}$ & $\mathrm{R}$ & O \\
\hline Cardiothoracic and vascular surgery & $\mathrm{R}$ & $\mathrm{R}$ & O \\
\hline Endocrinologist & $\mathrm{R}$ & $\mathrm{R}$ & $\mathrm{O}$ \\
\hline Epileptologist & $\mathrm{R}$ & O & $\mathrm{O}$ \\
\hline Ethics & $\mathrm{R}$ & $\mathrm{R}$ & $\mathrm{R}$ \\
\hline Gastroenterologist & $\mathrm{R}$ & $\mathrm{R}$ & O \\
\hline General surgeon & $\mathrm{R}$ & $\mathrm{R}$ & O \\
\hline Hematologist/oncologist & R & $\mathrm{R}$ & O \\
\hline Infectious disease & $\mathrm{R}$ & $\mathrm{R}$ & $\mathrm{O}$ \\
\hline Nephrologist & R & R & O \\
\hline Palliative care/supportive care medicine & $\mathrm{R}$ & $\mathrm{R}$ & O \\
\hline Pathologist & $\mathrm{R}$ & O & O \\
\hline Psychiatrist/psychologist & $\mathrm{R}$ & O & $\mathrm{O}$ \\
\hline Pulmonologist & R & $\mathrm{R}$ & O \\
\hline Radiologist & $\mathrm{R}$ & $\mathrm{R}^{*}$ & $\mathrm{R}^{*}$ \\
\hline \multicolumn{4}{|l|}{ Nursing staff } \\
\hline Director/Manager with NCC experience & $\mathrm{R}$ & $\mathrm{R}$ & $\mathrm{N} / \mathrm{A}$ \\
\hline Nurse to patient ratio $2: 1$, criteria for $1: 1$ & $\mathrm{R}$ & $\mathrm{R}$ & N/A \\
\hline \% nurses with specialty certification (e.g., SCRN, CNRN, NVRN) & $\mathrm{R}$ & O & O \\
\hline All nurses offered initial orientation, ongoing education, annual performance eval, ongoing competency assessment & $\mathrm{R}$ & $\mathrm{R}$ & $\mathrm{R}$ \\
\hline BLS, ACLS for all nurses & $\mathrm{R}$ & $\mathrm{R}$ & $\mathrm{R}$ \\
\hline ENLS certification or equivalent & $\mathrm{R}$ & R & $\mathrm{R}$ \\
\hline Knowledge and education should reflect general critical care and neurocritical care concepts & $\mathrm{R}$ & $\mathrm{R}$ & $\mathrm{R}$ \\
\hline Manager has master's degree or higher or subspecialty certification & $\mathrm{R}$ & O & O \\
\hline Neurocritical care competencies (including ED staff) & $\mathrm{R}$ & R & $\mathrm{R}$ \\
\hline Nurse-sensitive quality indicators should be monitored & $\mathrm{R}$ & $\mathrm{R}$ & $\mathrm{R}$ \\
\hline Nursing policies and procedure & $\mathrm{R}$ & $\mathrm{R}$ & $\mathrm{R}$ \\
\hline Orientation to ICU, NCC & $R$ & $\mathrm{R}$ & N/A \\
\hline
\end{tabular}

\section{Advanced Practice Provider (APP)}

Additional training in NCC completed and documented

Involved in rounding with NCC team

NCC Quality/Education-focused APP

$\begin{array}{lll}R & R & O \\ R & R & O \\ R & R & O\end{array}$

\section{Nursing skills}

Management of external ventricular drains and lumbar drains

Address psychosocial needs of family

Administer drugs

Administer fluids

Management of available advanced neurological monitoring

Management, troubleshooting patient monitors

\begin{tabular}{lll}
$R$ & $R$ & $R$ \\
$R$ & $R$ & $R$ \\
$R$ & $R$ & $R$ \\
$R$ & $R$ & $R$ \\
$R$ & $R$ & $O$ \\
$R$ & $R$ & $R$ \\
\hline
\end{tabular}


Table 2 continued

\section{Standards}

Level I Level II Level III

Recognize, interpret, record physiologic parameters

Respiratory care techniques (including mechanical ventilation)

Resuscitation, including ENLS certification or equiv.

Wound care of cranial and spinal post-operative patients

$\begin{array}{lll}R & R & R \\ R & R & R \\ R & R & R \\ R & R & R\end{array}$

\section{Pharmacist}

Pharmacist dedicated to NCC unit/team

$\begin{array}{lll}R & O & O \\ R & R & O \\ R & O & O \\ R & R & R \\ R & O & O \\ R & R & R \\ R & R & O \\ R & R & R \\ R & O & O\end{array}$

Pharmacist with expertise in neurocritical care

Pharmacist with residency training or equivalent in high-acuity area

Doctorate degree in pharmacy

BCCCP (preferred) or BCPS certification

Attend in-hospital neurocritical medical emergencies

Engaged in clinical rounding with team, daily medication review

Involved in hospital committees, quality improvement

Provide neuropharmacology training to other caregivers

$\begin{array}{lll}R & R & R \\ R & R & R \\ R & R & R \\ R & R & O\end{array}$

\section{Respiratory therapy}

Supervisor responsible for training RT staff, maintenance of equipment, and quality control/review

RT department supervise training

Therapist in-house $24 \mathrm{~h}$ day

Therapist in-house with neurocritical care expertise

\section{Other team members}

Biomedical technician

OT with expertise in neurologic patient population

PT with expertise in neurologic patient population

PT/OT/ST available 7 days a week

PT/OT/ST establish response times for new consultation

PT/OT/ST with special expertise in NCC

Radiology technician

Registered dietician or Certified Nutrition Support Clinician

Social worker and case manager with expertise in care of neurologic patient population

Spiritual care and support

ST with expertise in neurologic patient population

Unit clerk

\section{Hospital facilities and services}

\section{Emergency department:}

Staffed by physician $24 \mathrm{~h}$ day***

Helipad

Resuscitation area-capable of managing 2 patients at once

Resuscitation area_capable of managing at least 1 pt

Comprehensive blood bank, all components

Type and screen, cross match within $1 \mathrm{~h}$

\section{Radiology/diagnostic (perform/interpret):}

Angiography capabilities $24 \mathrm{~h}$ day

Continuous EEG

CT scan available $24 \mathrm{~h}$ day

Diagnostic and therapeutic endoscopy

EKG

$\begin{array}{lll}R & R & R \\ R & R & O \\ R & R & O \\ R & R & O \\ R & R & R \\ R & R & O \\ R & R & R \\ R & R & R \\ R & R & R \\ R & R & R \\ R & R & O \\ R & R & O\end{array}$

Fluoroscopy

MRI available $24 \mathrm{~h}$ day

Nuclear scanning

Portable radiograph

Radiation therapy

$\begin{array}{lll}R & R & O \\ O & O & O \\ R & O & O \\ R & R & R \\ R & R & R \\ R & R & R\end{array}$

Radian theray 
Table 2 continued

\begin{tabular}{|c|c|c|c|}
\hline Standards & Level I & Level II & Level III \\
\hline STATEEG & $\mathrm{R}$ & $\mathrm{R}$ & $\mathrm{O}$ \\
\hline Transcranial Doppler & R & 0 & 0 \\
\hline Transesophageal echocardiogram & $\mathrm{R}$ & $\mathrm{R}$ & $\mathrm{O}$ \\
\hline Transthoracic echocardiogram & $\mathrm{R}$ & $\mathrm{R}$ & R \\
\hline Ultrasonography & $\mathrm{R}$ & R & $\mathrm{R}$ \\
\hline \multicolumn{4}{|l|}{ Laboratory } \\
\hline ABG available within 15 min & $\mathrm{R}$ & $\mathrm{R}$ & $\mathrm{R}$ \\
\hline \multicolumn{4}{|l|}{ Available labs within $1 \mathrm{~h}$ : } \\
\hline CBC, plt, differential count & $\mathrm{R}$ & $\mathrm{R}$ & R \\
\hline Chemistry & $\mathrm{R}$ & $\mathrm{R}$ & $\mathrm{R}$ \\
\hline Clotting studies & R & $\mathrm{R}$ & $\mathrm{R}$ \\
\hline CSF cell count & R & $\mathrm{R}$ & R \\
\hline Platelet function testing & $\mathrm{R}$ & $\mathrm{R}$ & $\mathrm{R}$ \\
\hline UA & R & $\mathrm{R}$ & R \\
\hline
\end{tabular}

Labs available within $3 \mathrm{~h}$ :

\begin{tabular}{|c|c|c|c|}
\hline Ammonia & $\mathrm{R}$ & $\mathrm{R}$ & R \\
\hline Magnesium, phosphorus & $\mathrm{R}$ & $\mathrm{R}$ & R \\
\hline Osmolality & $\mathrm{R}$ & R & R \\
\hline Tox screen & $\mathrm{R}$ & $\mathrm{R}$ & R \\
\hline \multicolumn{4}{|l|}{ Labs available 24 h day: } \\
\hline Culture and gram stain & $\mathrm{R}$ & $\mathrm{R}$ & R \\
\hline \multicolumn{4}{|l|}{ Operating room: } \\
\hline Available within 30 min, 24 h day & $\mathrm{R}$ & $\mathrm{O}$ & $c$ \\
\hline Available within $60 \mathrm{~min}, 24 \mathrm{~h}$ day & $\mathrm{R}$ & $\mathrm{R}$ & $c$ \\
\hline Second OR available within $45 \mathrm{~min}, 24 \mathrm{~h}$ day & $\mathrm{R}$ & O & $c$ \\
\hline Renal replacement therapy including intermittent hemodialysis and continuous renal replacement therapy & $\mathrm{R}$ & $\mathrm{R}$ & c \\
\hline
\end{tabular}

\section{Pharmacy:}

$24 \mathrm{~h}$ day for all requests*

Bedside urgent drug list

\section{Neurorehabilitation internal or affiliated}

$\begin{array}{lll}R & R & R \\ R & R & R \\ R & R & O\end{array}$

\section{Physical facility (unit)}

Dedicated beds

Separate rooms:

\begin{tabular}{|c|c|c|c|}
\hline Conference room & R & R & R \\
\hline Family counseling room & $\mathrm{R}$ & R & $\mathrm{R}$ \\
\hline Leadership office space & $\mathrm{R}$ & $\mathrm{R}$ & $\mathrm{R}$ \\
\hline On call room & $\mathrm{R}$ & $\mathrm{R}$ & $\mathrm{R}$ \\
\hline Patients' personal effects storage (may be internal) & $\mathrm{R}$ & $\mathrm{R}$ & $\mathrm{R}$ \\
\hline Staff locker room & $\mathrm{R}$ & $\mathrm{R}$ & $\mathrm{R}$ \\
\hline Staff lounge & $\mathrm{R}$ & $\mathrm{R}$ & R \\
\hline \multicolumn{4}{|l|}{ Unit rooms, unit makeup } \\
\hline Clean utility room & $\mathrm{R}$ & R & $\mathrm{R}$ \\
\hline Clocks & $\mathrm{R}$ & R & R \\
\hline Computerized laboratory reporting or efficient equivalent & $\mathrm{R}$ & $\mathrm{R}$ & $\mathrm{R}$ \\
\hline Counter, cabinet space & $\mathrm{R}$ & $\mathrm{R}$ & $\mathrm{R}$ \\
\hline Easy, rapid access to head of bed & $\mathrm{R}$ & $\mathrm{R}$ & R \\
\hline Emergency equipment storage & $\mathrm{R}$ & $\mathrm{R}$ & $\mathrm{R}$ \\
\hline Handwashing facility & $\mathrm{R}$ & $\mathrm{R}$ & $\mathrm{R}$ \\
\hline Isolation capacity & $\mathrm{R}$ & $\mathrm{R}$ & $\mathrm{R}$ \\
\hline Medication station with drug refrigerator and locked narcotics cabinet & $\mathrm{R}$ & $\mathrm{R}$ & $\mathrm{R}$ \\
\hline Nourishment station & $\mathrm{R}$ & $\mathrm{R}$ & $\mathrm{R}$ \\
\hline
\end{tabular}


Table 2 continued

Standards
Patient privacy provision
Patient toilet
Soiled utility room
Staff toilet
Television, radios
Two or more compressed air outlets/bed
Two oxygen outlets/bed
Two vacuum outlets/bed
Building code or federal code conforming
Heating, ventilation, air conditioning
Fire safety
Electrical safety
Plumbing
Illumination

Portable equipment:

\begin{tabular}{|c|c|c|c|}
\hline Automated bed scale & $\mathrm{R}$ & $\mathrm{R}$ & $\mathrm{O}$ \\
\hline Automated blood pressure and monitoring technology & $\mathrm{R}$ & $\mathrm{R}$ & $\mathrm{R}$ \\
\hline Blood warmer & $\mathrm{R}$ & $\mathrm{R}$ & $\mathrm{R}$ \\
\hline Bronchoscope & $\mathrm{R}$ & $\mathrm{R}$ & $\mathrm{O}$ \\
\hline Defibrillator/cardioverter & $\mathrm{R}$ & $\mathrm{R}$ & $\mathrm{R}$ \\
\hline Difficult airway management equipment & $\mathrm{R}$ & $\mathrm{R}$ & R \\
\hline Doppler ultrasonography & $\mathrm{R}$ & $\mathrm{R}$ & $\mathrm{R}$ \\
\hline EKG machine & $\mathrm{R}$ & $\mathrm{R}$ & $\mathrm{R}$ \\
\hline EEG machine & $\mathrm{R}$ & $\mathrm{R}$ & $\mathrm{O}$ \\
\hline Emergency cart & $\mathrm{R}$ & $\mathrm{R}$ & $\mathrm{R}$ \\
\hline Emergency drugs & $\mathrm{R}$ & $\mathrm{R}$ & $\mathrm{R}$ \\
\hline Emergent surgical airway equipment & $\mathrm{R}$ & $\mathrm{R}$ & $\mathrm{R}$ \\
\hline Endotracheal intubation equipment & $\mathrm{R}$ & $\mathrm{R}$ & $\mathrm{R}$ \\
\hline Equipment for intracranial access, external ventricular drain placement and intraparenchymal pressure monitoring & $\mathrm{R}$ & $\mathrm{R}$ & O \\
\hline Heating/cooling blankets & $\mathrm{R}$ & $\mathrm{R}$ & $\mathrm{R}$ \\
\hline Infusion pumps & $\mathrm{R}$ & $\mathrm{R}$ & $\mathrm{R}$ \\
\hline Intraosseous access and/or emergency cut down trays & $\mathrm{R}$ & $\mathrm{R}$ & $\mathrm{O}$ \\
\hline Isolation cart & $\mathrm{R}$ & $\mathrm{R}$ & R \\
\hline Oral/nasal airways & $\mathrm{R}$ & $\mathrm{R}$ & $\mathrm{R}$ \\
\hline Oto-ophthalmoscope & $\mathrm{R}$ & $\mathrm{R}$ & $\mathrm{R}$ \\
\hline Point-of-care ultrasonography & $\mathrm{R}$ & $\mathrm{R}$ & $\mathrm{O}$ \\
\hline Procedure lamp (if adequate in room lighting not available) & $\mathrm{R}$ & $\mathrm{R}$ & $\mathrm{R}$ \\
\hline Suction machine (in addition to bedside) & $\mathrm{R}$ & $\mathrm{R}$ & $\mathrm{R}$ \\
\hline Thermometers & $\mathrm{R}$ & $\mathrm{R}$ & $\mathrm{R}$ \\
\hline Transport monitor & $\mathrm{R}$ & $\mathrm{R}$ & $\mathrm{R}$ \\
\hline Vascular access equipment & $\mathrm{R}$ & $\mathrm{R}$ & $\mathrm{R}$ \\
\hline \multicolumn{4}{|l|}{ Respiratory support equipment: } \\
\hline Air compressor & $\mathrm{R}$ & $\mathrm{R}$ & $\mathrm{R}$ \\
\hline Air-oxygen blenders & $\mathrm{R}$ & $\mathrm{R}$ & $\mathrm{R}$ \\
\hline Bag-valve-mask resuscitation devices & $\mathrm{R}$ & $\mathrm{R}$ & R \\
\hline Chest physiotherapy and suctioning & $\mathrm{R}$ & $\mathrm{R}$ & $\mathrm{R}$ \\
\hline Continuous oxygen analyzers with alarms & $\mathrm{R}$ & R & R \\
\hline Mechanical ventilators & $\mathrm{R}$ & $\mathrm{R}$ & R \\
\hline MRI compatible ventilator & $\mathrm{R}$ & $\mathrm{R}$ & $\mathrm{O}$ \\
\hline Non-invasive mechanical ventilators & $\mathrm{R}$ & $\mathrm{R}$ & $\mathrm{R}$ \\
\hline Oxygen tanks & $\mathrm{R}$ & $\mathrm{R}$ & R \\
\hline
\end{tabular}


Table 2 continued

\begin{tabular}{|c|c|c|c|}
\hline Standards & Level I & Level II & Level III \\
\hline Respired gas humidifiers & $\mathrm{R}$ & $\mathrm{R}$ & $\mathrm{R}$ \\
\hline Spirometers & R & $\mathrm{R}$ & $\mathrm{R}$ \\
\hline \multicolumn{4}{|l|}{ Continuous monitoring equipment: } \\
\hline EKG, heart rate & $\mathrm{R}$ & $\mathrm{R}$ & $\mathrm{R}$ \\
\hline Advanced hemodynamics: invasive or non-invasive including cardiac output & $\mathrm{R}$ & R & O \\
\hline Arrhythmia detection/alarm & $\mathrm{R}$ & $\mathrm{R}$ & $\mathrm{R}$ \\
\hline Brain tissue oxygen monitoring & $\mathrm{O}$ & $\mathrm{O}$ & $\mathrm{O}$ \\
\hline CBF & O & 0 & O \\
\hline Esophageal pressure & $\mathrm{R}$ & R & $\mathrm{O}$ \\
\hline ETCO2 monitor & $\mathrm{R}$ & $\mathrm{R}$ & $\mathrm{R}$ \\
\hline Intracranial pressure & $\mathrm{R}$ & $\mathrm{R}$ & O \\
\hline Microdialysis & O & O & O \\
\hline O2 monitors & $\mathrm{R}$ & R & $\mathrm{R}$ \\
\hline Respirations & $\mathrm{R}$ & $\mathrm{R}$ & $\mathrm{R}$ \\
\hline Systemic arterial pressure & $\mathrm{R}$ & $\mathrm{R}$ & $\mathrm{R}$ \\
\hline Temperature & $\mathrm{R}$ & R & $\mathrm{R}$ \\
\hline \multicolumn{4}{|l|}{ Research and training } \\
\hline \multicolumn{4}{|l|}{ Physician training: } \\
\hline Unit/beds in facility with accredited neurology residency program & O & O & NA \\
\hline Unit provides clinical rotation for neurocritical care & $\mathrm{O}$ & O & NA \\
\hline Fellowship program in NCC & $\mathrm{O}$ & $\mathrm{O}$ & NA \\
\hline ENLS certification or equivalent & $\mathrm{R}$ & $\mathrm{R}$ & $\mathrm{R}$ \\
\hline BLS/ACLS certification or equivalent & $\mathrm{R}$ & $\mathrm{R}$ & $\mathrm{R}$ \\
\hline Program participates in regional/national meetings related to NCC & $\mathrm{R}$ & $\mathrm{R}$ & O \\
\hline
\end{tabular}

\section{Unit personnel training:}

BLS training for nurses, respiratory therapists

ENLS certification or equiv for nurses

$\begin{array}{lll}R & R & R \\ R & R & R \\ R & R & R\end{array}$

Ongoing continuing education related to NCC

\section{Regional education:}

\section{Provide local/regional NCC education \\ Provide NCC educational outreach to the public}

\section{Prehospital care and interfacility transport}

Educational programs in stabilization and transportation for EMS personnel Integration/communication with EMS

Periodic review of EMS/transport protocols for NCC diseases

Transfer arrangement with Level 1 NCC Unit

Transfer arrangements with referral hospitals

$\begin{array}{lll}R & R & R \\ R & R & O\end{array}$

\section{Quality assessment}

Discharge planning

Monitor quality metrics

Morbidity/mortality review

Safety review

Utilization review

$\begin{array}{lll}R & R & R \\ R & R & R \\ R & R & R \\ N A & R & R \\ R & R & R \\ R & R & O \\ R & R & R \\ R & R & R \\ R & R & R \\ R & R & R\end{array}$

$A B G$ arterial Blood Gas, $A C L S$ advanced cardiac life support, $A P P$ advanced practice provider, $B C C C P$ board certified critical care pharmacist, $B C P S$ board certified pharmacotherapy specialist, $B L S$ basic life support, $C B C$ complete blood count, $C B F$ cerebral blood flow, CNRN certified neuroscience registered nurse, CSF cerebrospinal fluid, CT computed tomagraphy, ED emergency department, EEG electroencephalogram, EKG electrocardiogram, EMS emergency medical services, ENLS emergency neurologic life support, ETCO2 End Tidal CO2, $h$ hours, ICU intensive care unit, min minutes, MRI magnetic resonance imaging, NA not applicable NCC neuroscience critical care, NVRN neurovascular registered nurse, $O$ optional, OPPE ongoing professional practice evaluation, OR operating room, OT occupational therapist, PGY Post Graduate Year, plt platelet, $p t$ patient, PT physical therapist, R recommended, RT respiratory therapy, SCRN stroke certified registered nurse, ST speech therapist, UA Urinalysis

*Remote or real time

**Standard may be met by transfer agreement

***Physician support must be available if not on-site and services offered by LIP 
or helipad. Similarly, Level III units should be capable of transferring patients via ambulance and/or helipad. Organizations housing NCCUs should have diagnostic resources available, including radiology and laboratory services. (Table 2)

Each patient-care area should have adequate space for the patient and equipment necessary for the care of the patient, as well as outlets for electricity, oxygen, compressed air, and vacuum suction. The unit itself should also house a medication station and have ready-access to the pharmacy or satellite pharmacy. Clean and soiled utility rooms should be present within the unit for rapid access to and disposal of supplies required for patient care. Designated isolation areas should be available in the event of a NCCU patient with a communicable illness. Emergency equipment and emergency power outlets should be available, and all NCCUs should conform to appropriate codes for fire safety, wireless connectivity, plumbing, electricity, and ventilation.

\section{Personnel}

\section{Program Leadership}

A coordinated and cohesive interdisciplinary team is critical to the functioning of a high-quality neurocritical care unit. As such, the neurocritical care service at both a Level I and Level II unit should have a distinct administrative unit and financial support, with oversight by dedicated physician, nursing and hospital executive leadership. Physician, nursing and hospital executive leadership should meet regularly to formally evaluate service needs, address resource utilization, and assess outcomes and performance measures. This team, with the additional participation of members across disciplines, should be recognized as a standing committee within the hospital organization. Interdisciplinary committee membership should include representation from physicians, nurses, advanced practice providers (APP), clinical pharmacist, fellowship or training/education coordinator, respiratory therapists, case managers, social workers, nutrition services, rehabilitation services, and other disciplines as appropriate. The committee should be responsible for operational management of the unit and quality outcome monitoring. A Level III unit should also have service oversight by key physician, nursing, and executive leaders with regular meetings to evaluate service needs but functioning as a distinct administrative unit is optional.

\section{Medical Director}

A dedicated medical director for neurocritical care patients should be appointed at all levels, and a detailed description of professional responsibilities should be agreed upon in writing. Medical directors of Level I and
Level II NCCUs should complete formal advanced education in neurocritical care through fellowship training in a recognized neurocritical care fellowship program or have met prior grandfathering criteria for specialty certification. The medical director is recommended to complete neurocritical care subspecialty certification within 2 years of eligibility after accepting the position. The medical director should name a qualified physician to fulfill his or her duties when the director is unavailable, and this should be communicated to the interdisciplinary team. It is the responsibility of the director to oversee maintenance of a database of vital patient statistics, supervise quality assurance and improvement, ensure staff education, and oversee clinical competence for additional physician staff caring for NCC patients. In Level I units it is recommended that the Director also facilitate research efforts.

\section{Physician Staffing}

Several studies and a recent meta-analysis demonstrate that patients with severe neurologic illness have better outcomes when managed by a physician and interdisciplinary team with experience and expertise in neurocritical care. Neurointensivist-led interdisciplinary care is associated with decreased mortality, improved functional outcomes for a variety of diagnoses, and improved resource utilization $[3,4,8]$. Physicians providing neurocritical care to patients in both the Level I and Level II NCCU should have subspecialty training in NCC and either be eligible for or have subspecialty certification in neurocritical care and hold privileges to provide neurocritical care at their organization. Physicians in Level III units should demonstrate evidence of subspecialty training in NCC. Physicians should maintain the minimum number of continuing medical education annually in neurocritical care-related topics to maintain certification. Additionally, physicians providing neurocritical care at Level I or II units should provide 12 weeks of ICU service annually of which at least 6 weeks must be in the $\mathrm{NCCU}$, and undergo ongoing professional practice evaluation case review in neurocritical care with a frequency in accordance with hospital policy. Additional criteria to hold privileges in neurocritical care should be determined by hospital organizations.

Due to the acuity of patients receiving care in the NCCU at a Level I unit, a neurointensivist with neurocritical care privileges or designee should provide on-site care $24 \mathrm{~h}$ a day, 7 days a week. When not on-site, care may be delegated to another in-house provider. When care is delegated to other providers, the neurointensivist must be on call and available to return calls within $5 \mathrm{~min}$ and arrange for a physician or APP to be at the NCCU bedside or via telemedicine within $5 \mathrm{~min}$. 
The designee if the neurointensivist is not on-site may include a trainee physician or an APP (advanced practice nurse [APN] or physician's assistant [PA]) with specialized training in neurocritical care. Physicians-in-training may include a physician in an accredited neurocritical care fellowship, physicians in a fellowship with neurocritical care and cerebrovascular experience as determined and documented by the training program, or a resident physician in at least their second year of residency training who has completed focused training in neurocritical care. All trainee physicians and APPs designated to care for patients should maintain Emergency Neurologic Life Support (ENLS) certification or equivalent curriculum and complete additional focused training in neurocritical care. Such training should be clearly outlined and managed by program leadership.

At Level II units, 24-hour on-site care may be optional as patient acuity would not warrant this service. However, the neurointensivist should be available to return calls within $10 \mathrm{~min}$ and be available in-person at the NCCU bedside or via telemedicine within $30 \mathrm{~min}$ to manage acute situations. Level III units should have a certified neurointensivist available by telemedicine or telephone.

Additional medical subspecialists including a neurosurgeon, radiologist, neurointerventionalist, anesthesiologist, general surgeon, cardiothoracic and vascular surgeons, cardiologist, nephrologist, hematologist/oncologist, pulmonologist, endocrinologist, gastroenterologist, infectious disease specialist, pathologist, psychiatrist or psychologist, palliative/supportive care consultant, physiatrist, cerebrovascular neurologist, and epileptologist are recommended for Level I units. Please refer to Table 2 for recommendations for Level II and Level III units.

Additional subspecialty physician and interdisciplinary teams should be available for consultation as needed, and appropriate teams should be determined by program leadership according to common diagnoses and services provided within the program. This should include appropriate services to manage complications of neurocritical care illness.

\section{Nursing}

Nursing expertise and adequate staffing are important contributors to quality patient outcomes. A nurse manager or director with substantial critical care and neurosciences expertise should be designated for the NCCU. The manager/director should have specialty certification and/or a master's degree or higher in nursing or nursing administration. The nurse manager/director should work in collaboration with hospital administration, medical leadership and the interdisciplinary team to ensure a safe and high-quality environment for nursing practice.
This includes assuring appropriate staffing levels, nursing skill mix and resources are available to provide safe and quality care in the NCCU. All nurses working in the NCCU should be offered initial orientation, ongoing education, annual performance evaluation and ongoing competency assessment. The orientation, competency assessment and annual evaluation should include general critical care concepts. Initial orientation to critical care concepts includes either didactic or computer-based learning courses (e.g., American Association of CriticalCare Nurses Essential of Critical Care). Mandatory clinical certifications should include Basic and Advanced Life Support for adult units. The length of orientation should be based on whether nurses are new graduates or experienced. New graduate nurses should be part of a nurse residency program (6-12 months) where the complexities of managing critically ill patients and the opportunities for assessment, reflection and focused education occurs. Orientation for experienced nurses should be tailored to their experience and expertise. However, neurocritical care nursing is a specialty within critical care and requires an educational curriculum and competency assessment beyond only general critical care concepts. As such, nurses should receive focused initial and ongoing education and competency assessment for common neurocritical care concepts, monitoring, procedures and devices. This may include but is not limited to the ENLS curriculum, a program that outlines the key care priorities of 14 neurocritical care diagnoses in the first few hours of management. Supplemental information about the care of common neurocritical care diseases past the first few hours of management is necessary for all staff working in neurocritical care. Annual education offerings related to the neurologic disease process and patient management of neurocritically ill patients should be provided. Skill and competency of the nurses should be assessed periodically. Patient assignments should be based on the individual nurse's basic and advanced competencies. Nursing care of patients in the NCCU should be supported by evidence-based protocols, policies and procedures. Nurse-sensitive quality indicators (e.g., fall with injury, catheter associated urinary catheter infection, central line associated blood stream infection, pressure ulcer, mobility, etc.) and indicators that describe nursing care (e.g., skill mix, nurse/patient ratios, voluntary turnover) should be closely monitored by the NCCU leadership, benchmarked internally and externally and addressed if concerning trends are identified [9].

Staffing ratios in the NCCU should mirror the general critical care unit practice of 1 nurse to 2 patients (1:2), with the option for $1: 1$ staffing when acuity necessitates more focused nursing attention. Some states legislate minimum staffing requirements, and these should 
be followed accordingly. Patients may meet criteria for higher acuity for a variety of reasons, including but not limited to advanced cardiac, neurologic or dialysis devices, multiple titratable vasoactive infusions, acute intracranial hypertension, status epilepticus with ongoing seizure activity, acute spinal cord injury and neuromuscular diseases and other such diagnoses as listed in Table 1. The NCCU should develop a guideline to outline high-acuity diagnoses that may warrant 1:1 care according to local admission patterns and assessed nurse competency. The NCCU should use staff competency as a guide when assigning nurses to patients. Competency in neurocritical care nursing should be tracked and charge or lead nurses assigning patients should be confident the assignment matches the critical needs of the patient and the competency of the nurse.

Specialty certification in nursing allows nurses to demonstrate specialty knowledge in their area of practice and may be associated with improved patient outcomes [10]. Nursing specialty certification is increasingly being used as an indicator of high-quality nursing by hospital organizations and external regulatory bodies [11]. Nursing staff in the NCCU should be encouraged to seek specialty certification. At this time, there is no nursing certification focused solely on neurocritical concepts. However, certification as a critical care registered nurse, neuroscience registered nurse, stroke certified registered nurse, Advanced Neurovascular Practitioner, or neurovascular registered nurse certifications covers some, but not all, aspects of neurocritical care nursing.

\section{Advanced Practice Providers}

APP is a term that generally encompasses the roles of APNs (nurse practitioners (NP) and clinical nurse specialists (CNS), or as defined by the state board of nursing) and PA. APPs play a key role in the delivery of care to patients in the NCCU and should be viewed as critical team members. Common APN duties include providing clinical care to patients, expert nursing consultation, leading evidence-based practice and quality improvement initiatives, providing disease specific advanced education and leading or supporting research initiatives. The APN should hold a masters or doctorate degree in nursing and have expertise in the field of neurocritical care.

The educational preparedness and scope of practice differs between NP, CNS and PAs and may also vary according to state boards of medicine and nursing. However, all three roles are prepared at either the masters or doctoral level of education and cover a general curriculum rather than addressing subspecialties in depth. As such, additional training is neurocritical care is necessary for an APP to function autonomously as a provider in the NCCU. NP and CNS educational and certification curriculums offer either a primary care or acute care focus, and Adult Gerontology Acute Care NPs or CNSs are best prepared to provide care in the adult NCCU as outlined by the American Nurses Credentialing Center consensus model for Advanced Practice Registered Nurses education $[12,13]$.

When APPs provide direct patient care to the neurocritical care population, they should be provided a detailed orientation to neurocritical care assessment, diagnosis, management and procedures according to the needs of the patient population and organizational resources. They should be involved in rounding with the entire NCC team to enhance communication and seamless care. APPs should be credentialed in the procedures deemed necessary by the organization for the provision of care. Similar to nursing staff, new graduate APPs require focused training, and this may be done in a formal fellowship or informal manner by the program. Prior to providing independent care, the APP should be assessed for knowledge and competency in neurocritical care concepts. When multiple APPs provide care in the NCCU, they may benefit from a lead APP position who helps to coordinate and manage the team. APPs working with the medical team should be engaged in grand rounds, case review, morbidity and mortality review as well as focused and ongoing professional practice in neurocritical care.

\section{Pharmacy Services}

Pharmacists are essential members of the neurocritical care team. Intensive care pharmacist have demonstrated the ability to reduce medication costs [14-17], reduce adverse drug reactions/drug-drug interactions [18-21], decrease thromboembolic events [22], decrease ventilator days [23], improve morbidity, mortality and decrease length of ICU/hospital stay [22-25]. The only published study of pharmacists in the neurointensive care unit demonstrated a reduction in pharmacy acquisition cost from $\$ 4833$ to $\$ 3239$ per patient, a decrease in ICU length of stay (8.56 to 7.24 days, $\mathrm{p}=0.003$ ) and a significant decrease in readmission rates between the two groups $(\mathrm{P}<0.05)$ [15]. In the United States, The Joint Commission, The Society of Critical Care Medicine (SCCM), and The Joint Commission of Pharmacy Practitioners encourages pharmacists to practice higher level pharmacotherapy and further recommends the integration of a dedicated pharmacist into the ICU team to be accountable to manage all medication therapies [26, 27]. Other pharmacy organizations including American College of Clinical Pharmacy, and American Society of HealthSystem Pharmacist have proposed mandatory residency training (PGY1) for all new pharmacist entering a patient-care practice by the year 2020 [28]. Additional 
training opportunities for those who choose to care for critically ill patients include specialty (PGY2) residency in critical care, emergency medicine, transplant, or other higher acuity care area. For a pharmacist to successfully care for patients in the NCCU, a strong knowledge base and diverse skill set in both critical care and neurology is essential. Additionally, effective communication, critical thinking, leadership skills, and time management are all necessary qualities that a neurointensive care pharmacist must possess. We propose a list of essential, recommended, and optimal credentials that would prepare a pharmacist to care for patients with acute brain injury.

The essential credentials required are a doctorate in pharmacy degree, or equivalent outside the United States, with valid licensure that provides the basic knowledge, skill sets, and qualifications to function as an independent practitioner. Additionally, it is essential to complete one postgraduate year of residency to expose the clinician to a variety of clinical experiences and allow the practitioner to practice independently with mentorship. For those without the opportunity to complete a PGY1 residency, recommendations for experience equivalency should be a minimum of 5 years of experience with active direct patient-care services providing medication use management and medication educational services. Supplemental recommended neuropharmacotherapy training includes completion of the Pharmacotherapy of Neurocritical Care Series and ENLS certificate program. Additional recommended credentials included a specialty second year postgraduate residency (PGY2) in critical care, emergency medicine or other higher acuity care experience, Board of Pharmaceutical Specialist certification in Pharmacotherapy (BCPS), and Basic and Advanced Cardiac Life Support certification. Optimal certification would include all the above and includes Board of Pharmaceutical Specialties certification in Critical Care certification in lieu of BCPS certification and/or Advanced Practice Provider Certificate as regulated by each state.

Dedicated pharmacist coverage and 24-hour on call coverage by a critical care pharmacist is recommended for Level I units. Pharmacists caring for patients in the NCCU should be actively engaged in direct patient care daily with an active participation during bedside rounds and daily medication review and reconciliation. Pharmacists should attend medical emergencies in the hospital to assist in drug preparation and administration, as appropriate by state and hospital regulations.

Neuropharmacotherapy education to other healthcare providers is essential as well as being a self-directed learner to ensure evidence-based medicine is being practiced. Teaching and mentoring students and residents, including but not limited to pharmacy, nursing, and physicians, is recommended. Participation in development of national guidelines, writing tertiary publications, and leading the overall development of the field is optimal.

Involvement in hospital committees and engagement in quality improvement initiatives are each essential to ensure protocols and policies promote rational and safe medication use and stimulate multidisciplinary involvement. Contribution in either benchmark, translational, or clinical research is optimal.

\section{Respiratory Therapy Services}

Many patients in the NCCU present with or develop respiratory complications. Collaboration with a team of skilled respiratory therapists (RT) is essential. At all programs, RTs should be in-house $24 \mathrm{~h}$ per day. RTs in Level I and Level II units should have focused knowledge and experience managing patients with neurocritical care illness, and regular staff should be dedicated to the unit. Current evidence demonstrates that protocols and procedures for rapid weaning for extubation, tracheostomy management and oxygen titration driven by respiratory therapists (RTs) improve outcomes and cost-effectiveness [29]. At both Level I and II programs, protocols for these services should be developed by the NCC team in collaboration with RT leaders within the organization. Organizations should review NCCU acuity in relation to RT staffing and adjust accordingly. All RTs working with NCC patients should receive an orientation and competency assessment reflective of the acuity and patient population. The respiratory therapy department should supervise training, quality measures and equipment with support from the NCCU program leadership. Both Level I and Level II units should have MRI compatible ventilators.

\section{Physical, Occupational, and Speech and Language Therapy Services}

Physical Occupational and Speech and Language therapists are essential personnel in a NCCU program, and therapists should be available 7 days a week in both Level I and II units. Therapists in these units should have special expertise in the neurocritical care patient population. The program should establish reasonable expected response times for new patient consultations and staffing should reflect the patient acuity and therapy recommendations to allow for ongoing therapy 7-days a week as needed.

\section{Nutritional Support}

Registered dietitian nutritionists qualified to evaluate the complex relationship between illness and malnutrition in critically ill patients should be available for consultation 
daily in Level I and II units. Expertise and qualification is based on successful training as a specialty trained dietitian with or without advanced certifications [30, 31].

\section{Additional Support Services}

Delivery of high-quality care to neurocritically ill patients requires the efforts of multiple team members. These include unit clerks who facilitate communication between various members of the care team and patient families in addition to clerical responsibilities. Environmental and custodial service personnel facilitate room turnaround while maintaining optimal sanitary conditions for critically ill patients. Skilled and responsive information technology professionals are also essential to coordinating data acquisition and reporting. Spiritual care and other emotional support services are essential for the care of the patient and their loved ones. Social workers and case managers with expertise in the care of neurologic illness provide valuable information and facilitate in transitions of care.

\section{Training, Continuing Education, and Research}

The neurocritical care team should be responsible for the training of a multidisciplinary team of health care professionals across the institution. All health care providers working in the NCCU should routinely attend or participate in educational activities related to neurocritical care either at the institutional, local, regional or national levels.

A comprehensive neurocritical care program will be able to support access to sufficient patient volumes, educational opportunities and research in neurocritical care. Programs providing fellowship training must possess approval from the appropriate certifying bodies.

Nurses, respiratory therapists, and physicians must have Advanced Cardiac Life Support and neurological education such as ENLS certification or equivalent program in addition to further subspecialty training as delineated above. Physicians and fellowship trainees should in addition seek certification in advanced trauma life support or equivalent according to the patient populations served. Additionally, for all clinicians practicing NCC, participation in professional national and international organizations, including but not limited to the NCS, SCCM, or European Society of Intensive Care Medicine (ESICM) is optimal.

A set goal for continuous medical education credit is encouraged for the different team members. We propose $8 \mathrm{~h}$ annually of continuing education or equivalent nursing credit for all members of the multidisciplinary team. Core members defined as ICU director, associate director, fellowship director, nurse manager, CNS are encouraged to have $32 \mathrm{~h}$ of continuing education credits annually in subjects related to neurocritical care.
A comprehensive NCCU program should be able to should be able to demonstrate consistent research activity focused on all aspects of patient care including but not limited to disease management, patient outcome and quality research. Level I and Level II units should also provide educational outreach in NCC to the public.

Research is critical to advancing the understanding of neurocritical care illness, and the development of new strategies for patient management and improving outcomes. Level I units lead or participate in neurocritical care-related research. Level II units may also lead or participate in research, but a research program is not required.

\section{Medications}

There is a compendium of medications necessary to the management of the neurologically critically ill patient. All units at all levels should remain current with the available pharmaceuticals and maintain a pharmacy with the ability to rapidly respond to the management needs of this patient population. See Table 3 for a list of recommended essential medication categories that should be available on formulary at all Level I and II programs. Each organization should maintain its own policy and procedures around managing emergency medications for this patient population.

\section{Equipment}

The expansion of NCCUs nationwide is resulting in more tangible resources dedicated to patients with acute neurological injury. Essential equipment required for each patient includes ventilators and equipment to provide and monitor adequate respiratory support such as end tidal carbon dioxide monitors, delivery and monitoring systems for IV medications, and arterial and central venous access and hemodynamic monitoring equipment.

Imaging capabilities with computed tomography scan and ultrasonography should be available $24 \mathrm{~h}$ a day at all facilities. Magnetic resonance imaging and digital subtraction angiography capabilities should be available $24 \mathrm{~h}$ a day at Level I and Level II units.

Transthoracic echocardiogram should be available $24 \mathrm{~h}$ a day in all units, and Level I and Level II units should also be able to provide transesophageal echocardiogram.

Special equipment that is crucial in NCCUs includes electroencephalogram, duplex ultrasonography, and external ventricular drainage systems. Level I and Level II units should be capable of providing stat and continuous electroencephelogram monitoring, and Level I units should be able to provide transcranial Doppler ultrasonography. Equipment and protocols for multimodality monitoring may also be utilized in Level I units based on current guidelines and best clinical practice. Devices 
Table 3 Recommended rapidly available medication categories and examples

\begin{tabular}{|c|c|c|}
\hline Medication & Examples & Availability \\
\hline ACLS medications & Epinephrine, atropine, magnesium, sodium bicarbonate & $\leq 5$ min of STAT order \\
\hline Analgesic agents (IV push for acute analgesia) & Fentanyl, morphine, remifentanil, ketamine & $\leq 5 \mathrm{~min}$ of STAT order \\
\hline Antibiotics & Cefepime, ceftriaxone, vancomycin, cefazolin & $<15$ min of STAT order \\
\hline Antiepileptic agents & Phenytoin/fosphenytoin, levetiracetam, lacosamide, phenobarbital & $<15$ min of STAT order \\
\hline Antiepileptic agents (IV, IM, intranasal) & Benzodiazepine for emergent treatment of seizure & $\leq 5$ min of STAT order \\
\hline Antihypertensive agents & Nicardipine, clevidipine, labetalol, hydralazine & $\leq 5 \mathrm{~min}$ of STAT order \\
\hline Antiplatelet/anticoagulation & Heparin infusion, aspirin & $<15$ min of STAT order \\
\hline Cardiac rhythm and rate control agents & Adenosine, amiodarone, diltiazem, metoprolol & $\leq 5 \mathrm{~min}$ of STAT order \\
\hline Dextrose & D50\%, D10\% & $\leq 5$ min of STAT order \\
\hline Fibrinolytic & $\mathrm{tPA}$ & $\leq 5 \mathrm{~min}$ of STAT order \\
\hline Inotropic agents & Dobutamine, milrinone, epinephrine & $\leq 5 \mathrm{~min}$ of STAT order \\
\hline Osmotic agents & Mannitol, bolus dose hypertonic saline (23.4\%) & $\leq 5 \mathrm{~min}$ of STAT order \\
\hline Reversal agents & Prothrombin complex concentrate, naloxone, flumazenil & $\leq 5$ min of STAT order \\
\hline Sedative agents (IV push for acute sedation) & Benzodiazipine, propofol, etomidine & $\leq 5 \mathrm{~min}$ of STAT order \\
\hline Vasopressor agents & Norepinephine, phenylephine, epinephrine, dopamine, vasopressin & $\leq 5$ min of STAT order \\
\hline
\end{tabular}

$A C L S$ advanced cardiac life support, $I V$ intravenous, IM intramuscular

for targeted temperature management should be readily available as well.

\section{Processes}

\section{Protocols and Guidelines}

The NCCU team should develop and review on an annual basis multidisciplinary disease specific practice protocols or guidelines. The APP (CNS or NP) is well-suited to lead the development of the protocol working in a physician team framework. Critical steps include measuring current practice and outcomes, conducting a review of the literature, engaging members of the physician team and additional key stakeholders, building the protocol or guideline, educating the team, and evaluating/measuring the outcomes. Physician, ancillary, and nursing support from all levels is essential for success. Key policies surrounding prehospital care and interfacility transport, including transfer agreements with higher levels of care, should have the support of physician, nursing, and executive leadership at an institutional level. A pharmacist with neurocritical care expertise should be included in the development and review of any protocols or guidelines that involve medication management.

\section{Safety and Periodic Review}

ICU safety is an essential part of the foundation required to achieve high-quality health care. "Safe" care has been previously defined as care that minimizes risks and optimizes benefits [32]. Risks common to all ICU patients include medical errors, which are defined by the Institute of Medicine as diagnostic, treatment, nosocomial, procedural, and prophylactic. Building ICU infrastructure, staffing, and culture that prevent these errors, and encourages evidence-based best practices, is critical to ensuring ICU safety and high-quality care and is encouraged by multiple international organizations such as the SCCM, ESICM, Institute for Health Care Improvement (IHI), and Joint Commission [32-35]. Because the neurocritical care patient population includes highly time sensitive pathologies that may require the care of multiple subspecialists like neurointensivists, neurosurgeons, anesthesiologists, neurointerventionalists, and stroke specialists within the span of a few hours, the NCCU can be a challenging environment to ensure safe care.

Infrastructural elements like formal guidelines, protocols, care pathways, checklists, bundles, order sets, and other standardized processes of care, often facilitated by technology such as computerized order entry, bar code medication administration, and streamlined communication platforms, can help in reducing errors and promoting evidence-based care [35-38]. In addition to standardized processes that address basic ICU elements available from the aforementioned organizations, guidelines specific to neurocritical care disease states are available from the American Heart Association (AHA), NCS, American Academy of Neurology (AAN) [39], and Congress of Neurological Surgeons, among others.

In addition to standardized processes, it is also known that ICU staff's values, attitudes, perceptions, and commitment to safety, also known as safety culture, can affect patient outcomes [32, 34]. Several tools are available for assessing and improving an ICU's safety culture, such as the Hospital Survey on Patient Safety Culture (AHRQ, 2004), Patient Safety Climate Survey (IHI, 2006) and the Safety Climate Scale [40]. 
To track the efficacy of infrastructural elements and safety culture in a NCCU, periodic interdisciplinary review processes should be in place for both process (quality and safety) and outcomes measures. These measures may be created by the institution or unit, informed by the various measurement sets suggested by the IHI, University Health Consortium, SCCM, JC, AHA, and AAN, among others, or some combination thereof. These measures are ideally approached with similar rigor as good clinical research and require a data collection system that is accurate, comprehensive, consistent, and as automated as possible to ensure good data quality and sustainability [41]. Quality improvement initiatives can then be based off these data; embedding responsibility for them into the ICU's mission and specific job descriptions can be helpful in ensuring their continued optimization. In addition, creating a clear and easy to understand data reporting system (i.e., regularly updated dashboards) by which staff can understand data and historical trends is key to successful implementation of quality improvement initiatives [42]. Regular quality meetings, integration into peer review or morbidity and mortality conferences, or display of quality dashboards in areas highly trafficked by staff, are some ways to disseminate such data.

A quality improvement program is essential to assuring an ICU environment that is safe for the staff and for the patient. To implement a quality improvement program, evidence-based steps to implementing a quality improvement program include the following: identifying local resources, prioritizing projects, operationalizing measures and forming a business plan, performing an environmental scan, creating a data collection and reporting system, introducing behavior-changing strategies, and performing recurrent evaluation and maintenance [35]. A Comprehensive Unit-Based Safety Program is one validated approach that organizes the above elements and pairs them with accountability at the executive organizational level, and can lead to improved outcomes [43, 44].

\section{Conclusions}

In summary, neurocritical care is now a mature field which has reached a critical mass such that stratifying and structuring this subspecialized care has become necessary to deliver the best possible care and outcomes. This document provides an organizational framework for care delivery in highly resourced and lesser resourced communities in the United States. It is the goal of the NCS that structuring systems of care as outlined here will afford healthcare providers a framework from which to gather and coordinate resources, reduce unnecessary variation, and positively impact patient outcomes through delivery of high-quality and high value care.

\section{Author details}

${ }^{1}$ Cedars-Sinai Medical Center, Los Angeles, CA, USA. ${ }^{2}$ Rush University,

Chicago, IL, USA. ${ }^{3}$ Spectrum Health, Grand Rapids, MI, USA. ${ }^{4}$ Washington University, St. Louis, MO, USA. ${ }^{5}$ University of California, San Diego, San Diego, CA, USA. ${ }^{6}$ School of Medicine and Public Health, University of Wisconsin, Madison, WI, USA. ${ }^{7}$ Johns Hopkins, Baltimore, MD, USA. ${ }^{8}$ University of California, San Francisco, San Francisco, CA, USA. ${ }^{9}$ The Ohio State University, Columbus, OH, USA. ${ }^{10}$ The Queen's Medical Center, University of Hawaii, Honolulu, HI, USA.

\section{Acknowledgements}

We deeply appreciate the support and critical review of this document by the NCS Executive Committee, the NCS Quality Committee, and the NCS Guidelines Committee.

\section{Author Contribution}

All authors contributed to the conception, drafting, and critical revision of the manuscript.

\section{Source of support}

No grant support was received from any funding agency in the public, commercial, or not-for-profit sectors.

\section{Compliance with Ethical Standards}

\section{Conflict of interest}

AMM reports honoraria received from the Medical Speakers Network, serving on a Scientific Advisory Board for Portola Pharmaceuticals, and as an ENLS Instructor. SL reports personal fees from Stryker NV/SPS, Lombardi Hill, and Sarah Livesay LLC. TH reports personal fees from Cheisi and UCB Pharma. JM is a shareholder of iVMD and personal fees from CMTO.

Published online: 24 September 2018

\section{References}

1. Wijdicks EF. The history of neurocritical care. Handb Clin Neurol. 2017;140:3-14.

2. Dhar R, Rajajee V, Finley Caulfield A, et al. The state of neurocritical care fellowship training and attitudes toward accreditation and certification: a survey of neurocritical care fellowship program directors. Front Neurol. 2017;8:548

3. Suarez $\mathrm{Jl}$, Zaidat OO, Suri MF, et al. Length of stay and mortality in neurocritically ill patients: impact of a specialized neurocritical care team. Crit Care Med. 2004;32:2311-7.

4. Kramer AH, Zygun DA. Do neurocritical care units save lives? Measuring the impact of specialized ICUs. Neurocrit Care. 2011;14:329-33.

5. Harrison DA, Prabhu G, Grieve R, et al. Risk Adjustment In Neurocritical care (RAIN)-prospective validation of risk prediction models for adult patients with acute traumatic brain injury to use to evaluate the optimum location and comparative costs of neurocritical care: a cohort study. Health Technol Assess (Winchester, England). 2013; 17:vii-viii, $1-350$.

6. Rosenberg DI, Moss MM. Guidelines and levels of care for pediatric intensive care units. Pediatrics. 2004;114:1114-25.

7. Marshall JC, Bosco L, Adhikari NK, et al. What is an intensive care unit? A report of the task force of the World Federation of Societies of Intensive and Critical Care Medicine. J Crit Care. 2017;37:270-6.

8. Lott JP, Iwashyna TJ, Christie JD, Asch DA, Kramer AA, Kahn JM. Critical illness outcomes in specialty versus general intensive care units. Am J Respir Crit Care Med. 2009;179:676-83.

9. Brown DS, Donaldson N, Burnes Bolton L, Aydin CE. Nursing-sensitive benchmarks for hospitals to gauge high-reliability performance. J Healthcare Qual Off Publ Natl Assoc Healthcare Qual. 2010;32:9-17.

10. Fleischman RK, Meyer L, Watson C. Best practices in creating a culture of certification. AACN Adv Crit Care. 2011;22:33-49.

11. Leyden C. Certification as a quality initiative. J Nurs Adm. 2015;45:467-8. 
12. APRN Consensus Model. 2018. 2017. http://www.nursecredentialing.org/ Certification/APRNCorner. Accessed 2 Sept 2018.

13. The ACWG, Committee NCoSBoNAA. Consensus model for APRN regulation: licensure, accreditation, certification \& education 2008.

14. Montazeri M, Cook DJ. Impact of a clinical pharmacist in a multidisciplinary intensive care unit. Crit Care Med. 1994;22:1044-8.

15. Weant KA, Armitstead JA, Ladha AM, Sasaki-Adams D, Hadar EJ, Ewend MG. Cost effectiveness of a clinical pharmacist on a neurosurgical team. Neurosurgery. 2009;65:946-50.

16. Patel NP, Brandt CP, Yowler CJ. A prospective study of the impact of a critical care pharmacist assigned as a member of the multidisciplinary burn care team. J Burn Care Res Off Publ Am Burn Assoc. 2006;27:310-3.

17. Kane SL, Weber RJ, Dasta JF. The impact of critical care pharmacists on enhancing patient outcomes. Intensive Care Med. 2003;29:691-8.

18. Leape LL, Cullen DJ, Clapp MD, et al. Pharmacist participation on physician rounds and adverse drug events in the intensive care unit. JAMA. 1999;282:267-70.

19. Wang J, Dong M, Lu Y, Zhao X, Li X, Wen A. Impact of pharmacist interventions on rational prophylactic antibiotic use and cost saving in elective cesarean section. Int J Clin Pharmacol Ther. 2015;53:605-15.

20. Ng TM, Bell AM, Hong C, et al. Pharmacist monitoring of QTc intervalprolonging medications in critically ill medical patients: a pilot study. Ann Pharmacother. 2008;42:475-82.

21. Rivkin $\mathrm{A}$, Yin $\mathrm{H}$. Evaluation of the role of the critical care pharmacist in identifying and avoiding or minimizing significant drug-drug interactions in medical intensive care patients. J Crit Care. 2011;26(104):e1-6.

22. MacLaren R, Bond CA. Effects of pharmacist participation in intensive care units on clinical and economic outcomes of critically ill patients with thromboembolic or infarction-related events. Pharmacotherapy. 2009;29:761-8.

23. Marshall J, Finn CA, Theodore AC. Impact of a clinical pharmacistenforced intensive care unit sedation protocol on duration of mechanical ventilation and hospital stay. Crit Care Med. 2008;36:427-33.

24. MacLaren R, Bond CA, Martin SJ, Fike D. Clinical and economic outcomes of involving pharmacists in the direct care of critically ill patients with infections. Crit Care Med. 2008;36:3184-9.

25. Netzer G, Liu X, Shanholtz C, Harris A, Verceles A, Iwashyna TJ. Decreased mortality resulting from a multicomponent intervention in a tertiary care medical intensive care unit. Crit Care Med. 2011;39:284-93.

26. The Joint Commission announces the 2009 National Patient Safety Goals and requirements. Joint commission perspectives Joint Commission on Accreditation of Healthcare Organizations 2008;28:1, 11-5.

27. Rudis MI, Brandl KM. Position paper on critical care pharmacy services. Society of Critical Care Medicine and American College of Clinical Pharmacy Task Force on s. Crit Care Med. 2000;28:3746-50.

28. Murphy JE, Nappi JM, Bosso JA, et al. American College of Clinical Pharmacy's vision of the future: postgraduate pharmacy residency train ing as a prerequisite for direct patient care practice. Pharmacotherapy 2006;26:722-33.
29. Kollef MH, Shapiro SD, Silver P, et al. A randomized, controlled trial of protocol-directed versus physician-directed weaning from mechanical ventilation. Crit Care Med. 1997;25:567-74.

30. Russell M, Stieber M, Brantley S, et al. American Society for Parenteral and Enteral Nutrition (A.S.P.E.N.) and American Dietetic Association (ADA): standards of practice and standards of professional performance for registered dietitians (generalist, specialty, and advanced) in nutrition support. Nutr Clin Pract Off Publ Am Soc Parenter Enter Nutr. 2007;22:558-86.

31. The Evolving Role of the Critical Care Dietitian. Society of Critical Care Medicine, 2011. http://www.sccm.org/Communications/Critical-Conne ctions/Archives/Pages/The-Evolving-Role-of-the-Critical-Care-Dietitian. aspx. Accessed 12 Sept 2017.

32. Stockwell DC, Slonim AD. Quality and safety in the intensive care unit. Journal of intensive care medicine. 2006;21:199-210.

33. De Keyser J, Gdovinova Z, Uyttenboogaart M, Vroomen PC, Luijckx GJ. Intravenous alteplase for stroke: beyond the guidelines and in particular clinical situations. Stroke. 2007;38:2612-8.

34. Huang DT, Clermont G, Kong L, et al. Intensive care unit safety culture and outcomes: a US multicenter study. Int J Qual Health Care. 2010;22:151-61.

35. Checkley W, Martin GS, Brown SM, et al. Structure, process, and annual ICU mortality across 69 centers: United States critical illness and injury trials group critical illness outcomes study. Crit Care Med. 2014;42:344-56.

36. Dykes PC, Rozenblum R, Dalal A, et al. Prospective evaluation of a multifaceted intervention to improve outcomes in intensive care: the promoting respect and ongoing safety through patient engagement communication and technology study. Crit Care Med. 2017:45:e806-13.

37. Wick EC, Galante DJ, Hobson DB, et al. Organizational culture changes result in improvement in patient-centered outcomes: implementation of an integrated recovery pathway for surgical patients. J Am Coll Surg. 2015;221:669-77.

38. Guidelines for standards of care for patients with acute respiratory failure on mechanical ventilatory support. Task Force on Guidelines; Society of Critical Care Medicine. Critical Care Medicine 1991;19:275-8.

39. Rowe AS, Goodwin H, Brophy GM, et al. Seizure prophylaxis in neurocritical care: a review of evidence-based support. Pharmacotherapy. 2013;34:396-409.

40. Pronovost PJ, Weast B, Holzmueller CG, et al. Evaluation of the culture of safety: survey of clinicians and managers in an academic medical center. Qual Saf Health Care. 2003;12:405-10.

41. Curtis JR, Cook DJ, Wall RJ, et al. Intensive care unit quality improvement: a "how-to" guide for the interdisciplinary team. Crit Care Med. 2006:34:211-8.

42. Dodek PM, Heyland DK, Rocker GM, Cook DJ. Translating family satisfaction data into quality improvement. Crit Care Med. 2004;32:1922-7.

43. Pronovost $\mathrm{P}$, Weast $\mathrm{B}$, Rosenstein $\mathrm{B}$, et al. Implementing and validating a comprehensive unit-based safety program. J Patient Saf. 2005;1:33-40.

44. Pronovost PJ, Berenholtz SM, Goeschel C, et al. Improving patient safety in intensive care units in Michigan. J Crit Care. 2008;23:207-21. 